\title{
Modulation of miR-26a-5p and miR-15b-5p Exosomal Expression Associated with Clopidogrel-Induced Hepatotoxicity in HepG2 Cells
}

\begin{abstract}
Renata C. Costa de Freitas ${ }^{1}$, Raul H. Bortolin ${ }^{1}$, Mariana B. Lopes ${ }^{1}$, Letícia Tamborlin ${ }^{2}$, Letícia Meneguello ${ }^{3}$, Vivian N. Silbiger ${ }^{1}$, Rosario D. C. Hirata ${ }^{4}$, Mário H. Hirata ${ }^{4}$, Augusto D. Luchessi ${ }^{2,3}$ and André D. Luchessi ${ }^{\text {* }}$.

${ }^{1}$ Department of Clinical and Toxicological Analysis, Federal University of Rio Grande do Norte, Natal, Brazil, ${ }^{2}$ Laboratory of Biotechnology, School of Applied Sciences, University of Campinas, Limeira, Brazil, ${ }^{3}$ Post graduation in Biological Science, Institute of Biosciences, São Paulo State University (UNESP), Rio Claro, Brazil, ${ }^{4}$ Department of Clinical and Toxicological Analyses, School of Pharmaceutical Sciences, University of São Paulo, São Paulo, Brazil
\end{abstract}

\section{OPEN ACCESS}

Edited by:

Nicolau Beckmann, Novartis Institutes for BioMedical

Research, Switzerland

Reviewed by:

Baitang Ning

United States Food and Drug Administration, United States

Stephan Krähenbühl,

University of Basel, Switzerland

Camilla Stephens,

University of Málaga, Spain

*Correspondence:

André D. Luchessi

andre.luchessi@outlook.com

Specialty section:

This article was submitted to Cardiovascular and Smooth Muscle

Pharmacology,

a section of the journal

Frontiers in Pharmacology

Received: 30 August 2017 Accepted: 28 November 2017 Published: 12 December 2017

Citation:

Freitas RCC, Bortolin RH, Lopes MB,

Tamborlin L, Meneguello L,

Silbiger VN, Hirata RDC, Hirata MH, Luchessi $A D$ and Luchessi $A D$ (2017)

Modulation of miR-26a-5p and

miR-15b-5p Exosomal Expression Associated with Clopidogrel-Induced Hepatotoxicity in HepG2 Cells.

Front. Pharmacol. 8:906 doi: 10.3389/fphar.2017.00906
Clopidogrel is an essential antiplatelet drug used to prevent thrombosis complications associated with atherosclerosis. However, hepatotoxicity is a potential adverse effect related to clopidogrel therapy. Exosome-derived miRNAs may be useful for improved monitoring of drug response and hepatotoxicity risk. In the present study, the expression of several exosomal miRNAs (miR-26a-5p, miR-145-5p, miR-15b-5p, and miR-4701-3p) and cell-derived mRNA targets (PLOD2, SENP5, EIF4G2, HMGA2, STRADB, and TLK1) were evaluated in HepG2 cells treated with clopidogrel $(6.25,12.5,25,50$, and $100 \mu \mathrm{M})$ for 24 and $48 \mathrm{~h}$. Then, clopidogrel cytotoxicity was evaluated by analyzing DNA fragmentation and the cell cycle profile using flow cytometry. Differential expression of exosome-derived miRNAs and cell-derived mRNAs was analyzed by RT-qPCR. Exposure of HepG2 cells to high concentrations of clopidogrel (50 and $100 \mu \mathrm{M}$ ) for $24 \mathrm{~h}$ caused significant DNA fragmentation (17.6 and 44.4\%, respectively; $p<0.05$ ) and 48 $\mathrm{h}(26.8$ and $48.9 \%$, respectively; $p<0.05)$, indicating cellular toxicity. Upregulation of miR-26a-5p and downregulation of miR-15b-5p was observed in cells exposed to $100 \mu \mathrm{M}$ clopidogrel for 24 and $48 \mathrm{~h}$. The miR-26a-5p target mRNAs HMGA2, EIF4G2, STRADB, and SENP5 were downregulated in HepG2 cells following exposure to cytotoxic concentrations of clopidogrel (50 and $100 \mu \mathrm{M}$ ) for $24 \mathrm{~h}$, and HMGA2 levels remained low after $48 \mathrm{~h}$ of treatment. $T L K 1$, a target of miR-15b-5p, was downregulated by 50 and $100 \mu \mathrm{M}$ clopidogrel at $24 \mathrm{~h}$. In conclusion, our results suggest that exposure to high concentrations of clopidogrel modulates the expression of exosomal miR-26a-5p and miR-15b-5p and their target mRNAs in HepG2 cells. Dysregulation of these miRNAs maybe modulate the regulatory pathways involved in clopidogrel-induced liver injury.

Keywords: clopidogrel, microRNAs, hepatotoxicity, HepG2 cell line

\section{INTRODUCTION}

Clopidogrel is an antiplatelet therapy currently used for the prevention and treatment of thromboembolic complications and for delaying the progression of atherosclerosis (Fintel, 2012), a silent chronic vascular pathology that is a major cause of cardiovascular ischemic events (Badimon and Vilahur, 2014). 
Clopidogrel is a pro-drug that is activated in the liver by several cytochrome P450 enzymes (CYPs) to the active metabolite, 2-oxo-clopidogrel, which inhibits platelet aggregation by binding irreversibly to purinergic receptor P2Y, G-protein coupled, 12 (P2Y12 receptor) (Sangkuhl et al., 2010; Fitzgerald and Fitzgerald, 2013; Yang et al., 2015). It can also be hydrolyzed, independent of CYP activity, by hepatic esterases, mainly carboxylesterase 1 (CES1), which generates the inactive carboxylic acid metabolite (CAM) (Beitelshees et al., 2015). Paraoxonase 1 (PON1) is involved in the biotransformation of 2-oxo-clopidogrel into a thiol active metabolite, which has been reported to be toxic (Bouman et al., 2011). The patient response to clopidogrel is highly variable, sometimes resulting in an increased risk of thrombotic events and adverse effects, which are important clinical problems in cardiovascular medicine (Beitelshees et al., 2015; Yang et al., 2015).

The most common adverse effects of clopidogrel treatment include gastrointestinal disorders, bleeding, thrombotic thrombocytopenic purpura, neutropenia, and aplastic anemia (Ochoa-Cortes et al., 2014; Kapila et al., 2015). Hepatotoxicity is a rare but potentially serious adverse effect related to clopidogrel treatment that can lead to fulminant hepatitis (Goyal et al., 2009; Monteiro et al., 2011; Zahno et al., 2013). However, the mechanisms of clopidogrel-associated hepatotoxicity have not been fully elucidated.

Advanced molecular analyses, particularly those used to measure gene expression, are useful for evaluating the changes related to organ dysfunction caused by adverse drug reactions, as characterized by the DNA damage that occurs during apoptosis (Vickers et al., 2017). In this way, the expression levels of various miRNAs have been proposed as novel biomarkers of druginduced hepatotoxicity (Fontana, 2014; Weiler et al., 2015; Hayes and Chayama, 2016).

miRNAs are short, non-coding RNAs of 18-25 nucleotides that are partially complementary to target mRNAs. miRNAs play a crucial role in the post-transcriptional regulation of gene expression, mostly through gene silencing, as miRNA binding suppresses the translation of target mRNAs and/or promotes their degradation (Stroynowska-Czerwinska et al., 2014; Hayes and Chayama, 2016).

miRNAs are found in both intracellular and extracellular environments (circulating miRNAs). They are also detected in exosomes, which are $40-100 \mathrm{~nm}$ vesicles that are released from many types of cells into the extracellular space and are involved in cell-cell communication (Huang et al., 2013; Zhang et al., 2015). Exosomes also play an important role in the early release of miRNAs during drug-induced liver injury (Bala et al., 2012; McGill and Jaeschke, 2015).

Some studies have evaluated the role of miRNAs in the clopidogrel response. One study showed that platelet expression of miRNAs, such as miR-26a, was associated with clopidogrel resistance in patients who underwent coronary stenting (Chen et al., 2016). Platelet miRNAs were also proposed as potential predictors of reduced response to clopidogrel in acute coronary syndrome (Sunderland et al., 2017).

We previously used bioinformatics tools to investigate the association of several miRNAs and target mRNAs with the response to clopidogrel. We showed that five miRNAs, miR-1455p, miR-26a-5p, miR-107, miR-15b-5p, and miR-4701-3p, were shown to influence platelet reactivity, clopidogrel response, and drug-induced toxicity, particularly hepatotoxicity (Freitas et al., 2016). This integrated analysis also showed that SENP5, EIF4G2, HMGA2, STRADB, and TLK1, which are potential targets of miR-26a and miR-15b, are associated with clopidogrel-induced hepatotoxicity (Freitas et al., 2016). PLOD2 was also described as a potential target of miR-26a in a model of bladder cancer (Miyamoto et al., 2016).

In this study, we investigated the effect of cytotoxic concentrations of clopidogrel on the expression of miR-145, miR-26a, miR-4701, and miR-15b in exosomes and their target mRNAs in HepG2 cells.

\section{MATERIALS AND METHODS}

\section{Cells and Culture}

HepG2 cells were obtained from the Rio de Janeiro Cell Bank (Rio de Janeiro, Brazil) and maintained in RPMI-1640 medium ( $\mathrm{pH}$ 7.4) supplemented with L-glutamine ( $2 \mathrm{mM}$, penicillin $(100 \mathrm{U} / \mathrm{mL})$, streptomycin $(100 \mu \mathrm{g} / \mathrm{mL})$, and $5 \%$ exosomedepleted fetal bovine serum. The cells were grown in cell culture flasks at $37^{\circ} \mathrm{C}$ in a humidified atmosphere containing $5 \% \mathrm{CO}_{2}$ to 80-90\% confluence.

\section{Treatment of HepG2 Cells with Clopidogrel}

For flow cytometry analysis, HepG2 cells were seeded in 24-well plates $\left(1.5 \times 10^{5}\right.$ cells/well $)$ and maintained in culture medium for $24 \mathrm{~h}$. Then, the cells were treated with 0.0 (vehicle), 6.25, $12.5,25,50$, and $100 \mu \mathrm{M}$ clopidogrel (Sigma-Aldrich, St. Louis, MO, USA) dissolved in dimethylsulfoxide (DMSO) at a final concentration of $0.1 \%$ for 24 and $48 \mathrm{~h}$.

For the miRNA and mRNA expression analyses, HepG2 cells were seeded in $150 \mathrm{~cm}^{2}$ plates $\left(9 \times 10^{6}\right.$ cells/plate $)$ and maintained in culture medium for $24 \mathrm{~h}$. Then, the cells were treated for 24 and $48 \mathrm{~h}$ with 0.0 (vehicle), 6.25, 12.5, 25, 50, and $100 \mu \mathrm{M}$ clopidogrel dissolved in DMSO at final concentration of $0.1 \%$.

\section{Analysis of Clopidogrel Cytotoxicity by Flow Cytometry}

DNA fragmentation and the cell cycle were analyzed by flow cytometry. HepG2 cells exposed to clopidogrel were collected by trypsinization, centrifuged at $200 \times \mathrm{g}$ for $5 \mathrm{~min}$ at room temperature $\left(\sim 25^{\circ} \mathrm{C}\right)$ and washed with $500 \mu \mathrm{L}$ of PBS. Cell pellets were fixed with $500 \mu \mathrm{L}$ of $70 \%$ (v/v) cold ethanol. Fixed cells were washed with PBS and then resuspended in $500 \mu \mathrm{L}$ of propidium iodide (PI) solution $(20 \mu \mathrm{g} / \mathrm{mL}$ of PI, $0.1 \%$ Triton X100 , and $10 \mu \mathrm{g} / \mathrm{mL}$ DNAse free RNAse in PBS) and incubated for $30 \mathrm{~min}$ in the dark.

Flow cytometry analysis was carried out using a BD Accuri ${ }^{\mathrm{TM}}$ C6 Plus Cytometer (BD Bioscience, San Jose, CA, USA). Tenthousand events were evaluated in each sample test. Data were collected from three independent experiments, performed in triplicate. Cells displaying hypodiploid DNA content (sub-G1) were marked as apoptotic. 
Cell supernatants were used to measure the levels of alanine transaminase (ALT) and aspartate transaminase (AST), two markers of liver injury, by colorimetric-enzymatic methods using a biochemical analyzer (BIO-2000 IL; Bioplus Products for Laboratories, Sao Paulo, Brazil).

\section{RNA Extraction from Exosomes and HepG2 Cells}

Exosomes were isolated from the supernatant of HepG2 cells treated with clopidogrel $(12.5,25,50$, and $100 \mu \mathrm{M})$ or vehicle (control) using the exoRNeasy Serum/Plasma Maxi kit (Qiagen, Hilden, Germany; Cat. Number: 77064), according to the manufacturer's recommendations. Briefly, pre-filtered supernatants from treated cells were mixed 1:1 with binding buffer and added to an exoEasy membrane affinity column to allow the exomes bind to the membranes. The columns were centrifuged at $500 \times \mathrm{g}$ for $1 \mathrm{~min}$ at room temperature $\left(\sim 25^{\circ} \mathrm{C}\right)$, and washed with washing buffer to remove non-specifically retained materials.

The exosomes were lysed by adding QIAzol (Qiagen) to the columns, and then the lysates were collected by centrifugation (Enderle et al., 2015). The Caenorhabditis elegans miR-39 (cel-miR-39), which is the Spike-In Control contained in the miRNeasy Serum/Plasma Kit (Qiagen; Cat. Number: 219610) was added to monitor RNA recovery and reverse transcription efficiency. RNA was quantified and purity was assessed by spectrophotometry using a Nanodrop ND-1000 (Thermo Scientific, Wilmington, DE, USA).

Total RNA was extracted from clopidogrel-treated HepG2 cells using TRIzol reagent (Invitrogen, Carlsbad, CA, USA) according to the manufacturer's protocol. RNA was quantified and purity was assessed by spectrophotometry using a Nanodrop ND-1000.

\section{Exosomal miRNA Expression by RT-qPCR}

The cDNA of the miRNAs was synthesized with the miScript II RT Kit (Qiagen; Cat. Number: 218161) according to the manufacturer's protocol using a Veriti ${ }^{\mathrm{TM}}$ 96-Well Thermal Cycler (Applied Biosystems, Carlsbad, CA, USA).

RT-qPCR analysis was performed with pre-validated miScript ${ }^{\circledR}$ Primer Assays (Qiagen; Cat. Number: 218300) for the following miRNAs: Hs_miR-26a_2 (MIMAT0000082), Hs_miR145_1 (MIMAT0000437), Hs_miR-15b_2 (MIMAT0000417), Hs_miR-4701-3p_1 (MIMAT0019799), and Hs_miR-107_2 (MIMAT0000104) using the miScript SYBR Green PCR Kit (Qiagen; Cat. Number: 218073). These miRNAs were select based on the results of our previous in silico study (Freitas et al., 2016).

RT-qPCR assays were carried out in 96-well plates using a 7500 Fast Real-Time PCR System (Applied Biosystems). The amplification program consisted of 40 cycles of $94^{\circ} \mathrm{C}$ for $15 \mathrm{~s}$, $55^{\circ} \mathrm{C}$ for $30 \mathrm{~s}$, and $70^{\circ} \mathrm{C}$ for $30 \mathrm{~s}$. Data were collected from three independent experiments performed in duplicate. miRNAs with Ct-values $<37$ were excluded from the statistical analysis.

miRNA expression was normalized to relative quantities (NRQs) in three steps, as previously described (Marabita et al., 2016): (i) the miRNA target Ct-values were scaled with cel-miR-39 (formula: RQ = miRNA target Ct - cel-miR-39 Ct); (ii) the geometric RQ means of all expressed miRNAs per sample were obtained to calculate the normalization factor (NF); and (iii) the NRQ-values were calculated using the formula: NRQ = miRNA target RQ/NF. The results are shown as the fold change (FC), which was calculated using the NRQ-values of clopidogreltreated cells $(6.25,12.5,25,50$, and $100 \mu \mathrm{M})$ divided by NRQsvalues of control cells (vehicle only). The NRQ-values obtained in the experiments are shown in Supplementary Table 2.

\section{RT-qPCR Analysis of mRNA Expression in HepG2 Cells}

The cDNA was synthesized with $2 \mu \mathrm{g}$ of total RNA using the SuperScript ${ }^{\circledR}$ III First-Strand Synthesis System (Invitrogen), according to the manufacturer's protocol using a Veriti ${ }^{\mathrm{TM}}$ 96Well Thermocycler (Applied Biosystems).

RT-qPCR was carried out in 96-well plates using primers specific for PLOD2, SENP5, EIF4G2, HMGA2, STRADB, TLK1, and GAPDH (Supplementary Table 1) and the QuantiTect SYBR Green PCR Kit (Qiagen; Cat. Number: 204145). The qPCR assays were carried in a 7500 Fast Real-Time PCR System (Applied Biosystems). The amplification program consisted of 40 cycles of $94^{\circ} \mathrm{C}$ for $15 \mathrm{~s}, 55^{\circ} \mathrm{C}$ for $30 \mathrm{~s}$, and $70^{\circ} \mathrm{C}$ for $30 \mathrm{~s}$. Data were collected from three independent experiments performed in duplicate. mRNAs with Ct-values $<37$ were excluded from the statistical analysis.

GAPDH, $18 S$ rDNA, and ACTB were used as reference genes. According to the NormFinder algorithm, GAPDH was the most stable gene under our experimental conditions, thus it was used as the endogenous control. Relative expression was calculated by the $2^{-\Delta \Delta \mathrm{Ct}}$ method (Livak and Schmittgen, 2001), using vehicle $(0 \mu \mathrm{M}$ clopidogrel $)$ treated cells as the control. The delta Ctvalues obtained in the experiments are shown in Supplementary Table 3.

Previously published studies and data available at the National Center for Biotechnology Information (NCBI PubMed; https:// www.ncbi.nlm.nih.gov/pubmed/) for indicated the regulatory pathway of miRNAs and their differently expressed mRNA targets were used.

\section{Statistical Analysis}

The statistical analysis was carried out using SPSS ${ }^{\circledR} 20$ software (IBM, New York, NY, USA). DNA fragmentation data are shown as means and standard error of the mean (SEM), and miRNA and mRNA expression data are shown as the fold change between clopidogrel-treated $(6.25,12.5,25,50$, and $100 \mu \mathrm{M})$ and control (vehicle only, $0 \mu \mathrm{M}$ clopidogrel) cells. The relative expression of the miRNAs and mRNAs in clopidogrel-treated cells were compared to those in control cells (vehicle) by Kruskal-Wallis ANOVA, and pairwise comparisons were performed with the Mann-Whitney test. A $p<0.05$ was considered statistically significant.

\section{RESULTS}

Toxic Effects of Clopidogrel in HepG2 Cells To verify the toxic effects of clopidogrel in a human liver cell line, HepG2 cells were treated with different concentrations of clopidogrel $(6.25,12.5,25,50$, and $100 \mu \mathrm{M})$ for 24 and $48 \mathrm{~h}$. 
Flow cytometry analysis showed that cells treated with 50 and $100 \mu \mathrm{M}$ clopidogrel showed a higher percentage of cells with fragmented DNA than control cells at $24 \mathrm{~h}$ (17.6 and $44.4 \%$, respectively; $p<0.05)$ and $48 \mathrm{~h}(26.8$ and $48.9 \%$, respectively; $p<0.05$; Figure 1). In addition, the cell cycle profile of these cells showed general damage following clopidogrel treatment (Figure 2). These results suggest that clopidogrel, at 50 and $100 \mu \mathrm{M}$, is potentially toxic to HepG2 cells. In contrast, cells treated with $6.25,12.5$, and $25 \mu \mathrm{M}$ clopidogrel did not show significant DNA fragmentation at $24 \mathrm{~h}(3.7,3.4$, and $4.8 \%$, respectively) or $48 \mathrm{~h}(2.3,3.1$, and $2.7 \%$, respectively) when compared to the levels in control cells $(2.3$ and $2.2 \%$ at 24 and $48 \mathrm{~h}$, respectively; $p>0.05$ ). These lower concentrations of clopidogrel also did not affect the cell cycle profile, indicating that these are non-toxic concentrations for HepG2 cells.
Analysis of AST and ALT activities in HepG2 cell supernatants showed similar values for clopidogrel-treated $(12.5,25,50$, and $100 \mu \mathrm{M})$ and control cells at 24 and $48 \mathrm{~h}(p>0.05$; Supplementary Figure 1).

\section{Expression of Exosome-Derived miRNAs}

The relative expression levels (fold changes) of exosome-derived miRNAs in the supernatants of HepG2 cells treated with different concentrations of clopidogrel are shown in Figure 3. After 24 and $48 \mathrm{~h}$ of exposure to $100 \mu \mathrm{M}$ clopidogrel, exosome miR-26a was upregulated (two-fold) and miR-15b was downregulated (fourfold) when compared to the levels in the control $(p<0.05)$. In contrast, the expression of miR-145 and miR-4701 in HepG2derived exosomes was not significantly influenced by clopidogrel treatment $(p>0.05)$. miR-107 was not further analyzed due to having a Ct-value above 37 .

\section{DNA fragmentation}

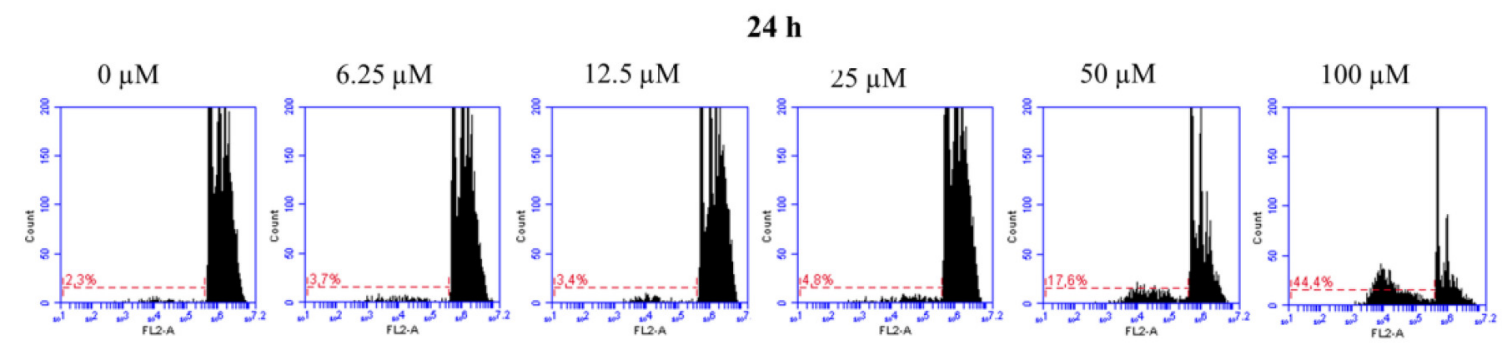

$48 \mathrm{~h}$
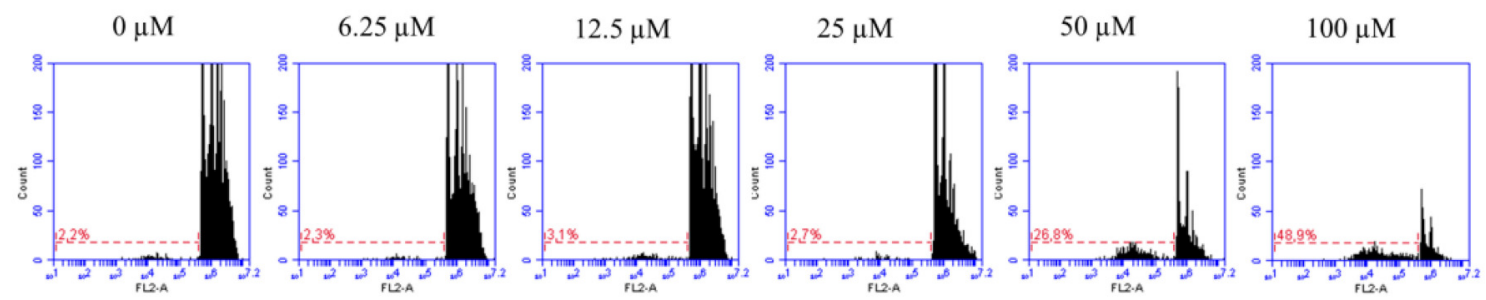

$24 \mathrm{~h}$

$48 \mathrm{~h}$
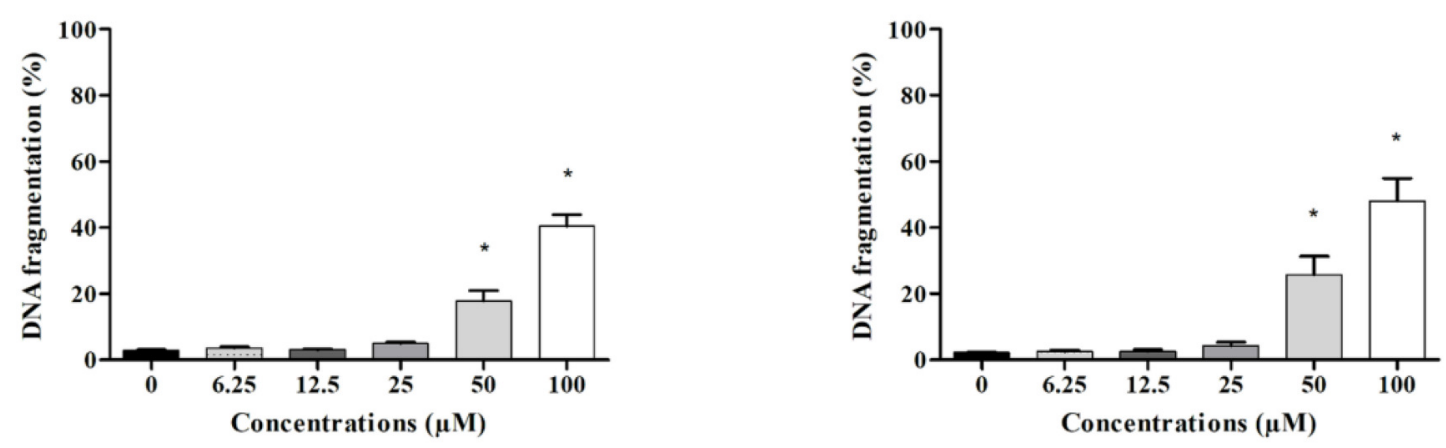

FIGURE 1 | DNA fragmentation in HepG2 cells treated with clopidogrel. Representative histograms and percentage of cells with fragmented DNA after treatment with clopidogrel $(6.25,12.5,25,50$, and $100 \mu \mathrm{M})$ for 24 and $48 \mathrm{~h}$. Data are shown as mean \pm SEM of three independent experiments performed in triplicate and compared by Kruskal-Wallis ANOVA and pairwise comparisons by Mann-Whitney test. ${ }^{*} p<0.05$ compared to control (vehicle, $0 \mu \mathrm{M}$ clopidogrel). 


\section{Cell Cycle}

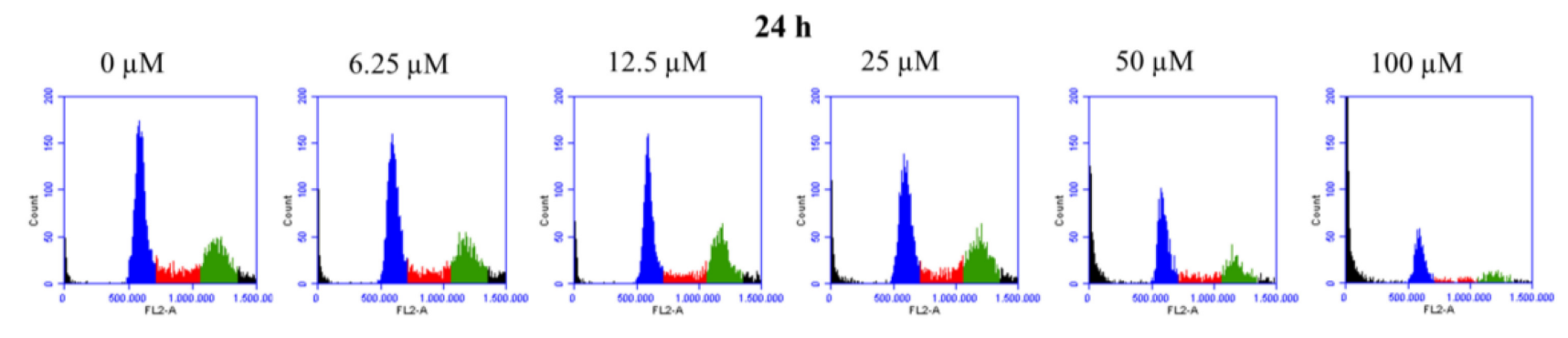

$48 \mathrm{~h}$
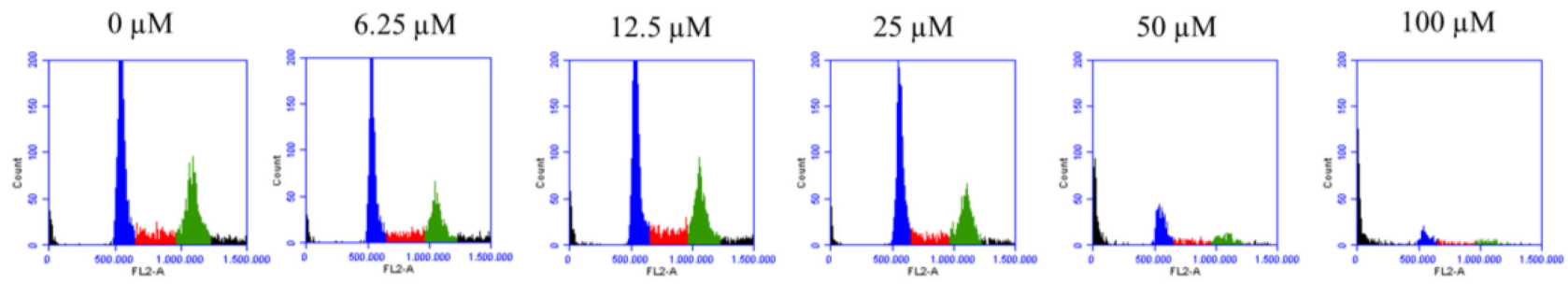

FIGURE 2 | Cell cycle profile of HepG2 cells treated with clopidogrel. Representative histograms of cells treated with clopidogrel (6.25, $12.5,25,50$, and 100 $\mu$ M) for 24 and 48 h. Blue: cells in the G0/G1 phase; Red: cells in the S phase; Green: cells in the G2/M phase. Data from 3 independent experiments performed in triplicate.

\section{Expression of HepG2 Cell-Derived mRNAs}

The relative expression of cell-derived mRNAs in cells treated with different concentrations of clopidogrel after 24 and $48 \mathrm{~h}$ is shown in Figures 4, 5, respectively. EIF4G2, HMGA2, STRADB, SENP5, and TLK1 levels in HepG2 cells were two-fold lower after $24 \mathrm{~h}$ of exposure to cytotoxic concentrations of clopidogrel (50 and $100 \mu \mathrm{M})$ than the levels in control cells $(p<0.05$; Figure 4). SENP5 mRNA levels were also reduced in cells treated with non-cytotoxic concentrations of clopidogrel (12.5 and $25 \mu \mathrm{M})$ for $24 \mathrm{~h}(p<0.05)$ when compared to the levels in control cells. Conversely, PLOD2 mRNA expression did not vary in cells treated with any concentration of clopidogrel for $24 \mathrm{~h}$.

After $48 \mathrm{~h}$ of clopidogrel treatment, HMGA2 expression remained downregulated in cells treated with cytotoxic concentrations of clopidogrel $(50$ and $100 \mu \mathrm{M})$ when compared to the expression in the control ( $p<0.05$; Figure 5). However, the mRNA levels of EIF4A2, PLOD2, STRADB, SENP5, and $T L K 1$ were not altered by $48 \mathrm{~h}$ of treatment with clopidogrel $(p>0.05)$.

The regulatory pathways associated with these differentially expressed exosome-derived miRNAs and their target mRNAs are shown in Table 1. The regulatory pathways for miR-26a were liver regeneration, cell cycle control, and apoptosis. The targets of miR-26a, HMGA2, EIF4G2, STRADB, and SENP5, are involved in cell growth and development, cell cycle control, DNA damage, and apoptosis. miR-15b is involved in cell survival and cell cycle control, and its target, TLK1, is associated with cell cycle control.

\section{DISCUSSION}

In this study, the toxic effects of clopidogrel treatment on exosomal miRNAs and their cellular target mRNAs were evaluated in HepG2 cells. Exposure to high concentrations of clopidogrel $(50$ and $100 \mu \mathrm{M})$ led to fragmented DNA in a large number of cells as well as general damage as observed by the altered cell cycle profile of clopidogrel-treated HepG2 cells, which are suggestive of clopidogrel cytotoxicity. Previous studies suggested that the levels of clopidogrel in the liver of patients treated with a maintenance dose of $75 \mathrm{mg}$ per day may reach $10 \mu \mathrm{M}$ (Caplain et al., 1999; Zahno et al., 2010, 2013). Likewise, 50 and $100 \mu \mathrm{M}$ clopidogrel are equivalent to the loading standard dose $(300 \mathrm{mg})$ and high dose $(600 \mathrm{mg})$ prescribed for percutaneous coronary intervention (angioplasty) protocols.

In a previous study, 10 and $100 \mu \mathrm{M}$ clopidogrel were shown to be toxic to primary human hepatocytes when combined with rifampicin (an inducer of CYP3A4) and to HepG2 cells overexpressing CYP3A4 (Zahno et al., 2013). In addition, high concentrations of clopidogrel $(100$ and $300 \mu \mathrm{M})$ were significantly toxic to primary rat hepatocytes and HepG2 cells coincubated with recombinant CYP2B6 and CYP2C19 (Zhai et al., 2016). These effects have been attributed to 2-oxo-clopidogrel, a bioactive metabolite, produced by CYP enzymes (Maffrand, 2012; Zhai et al., 2016). In contrast, it has been shown that high concentration $(100 \mu \mathrm{M})$ of clopidogrel and its major inactive metabolite (CAM) are not toxic to wild-type HepG2 cells (Zahno et al., 2013). The lack of clopidogrel toxic effect on HepG2 cells 
$24 \mathrm{~h}$

miR-26a

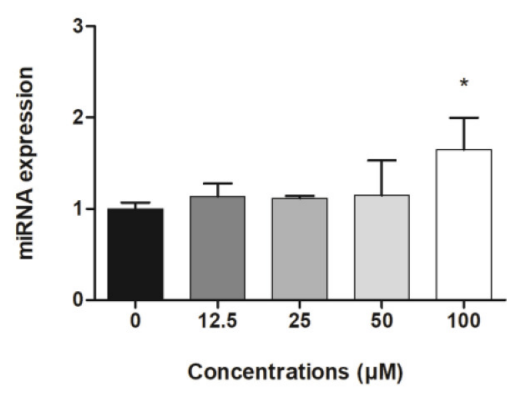

miR-15b

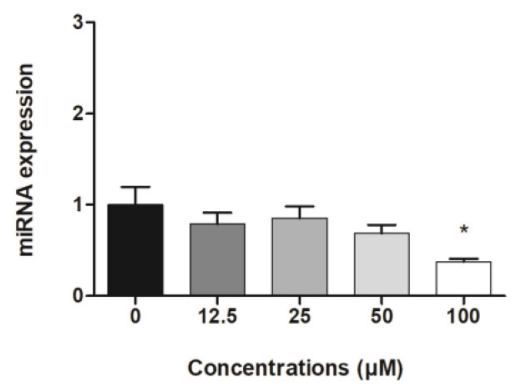

miR-4701

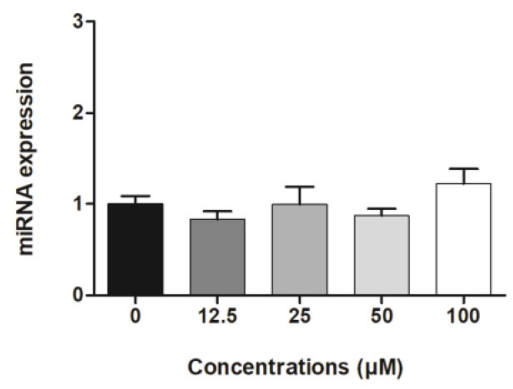

$\operatorname{miR}-145$

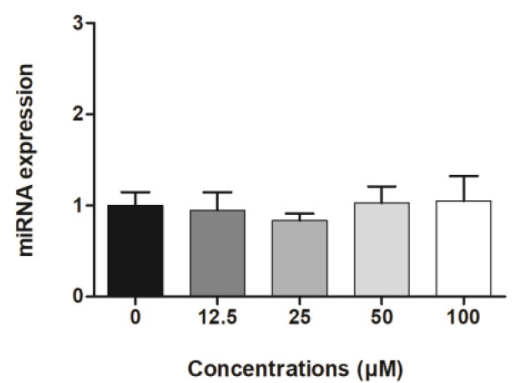

$48 \mathrm{~h}$

miR-26a

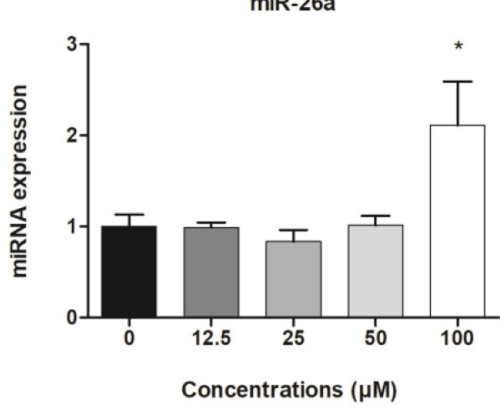

miR-15b

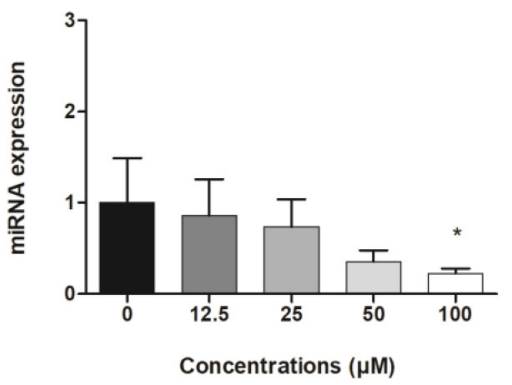

miR-4701

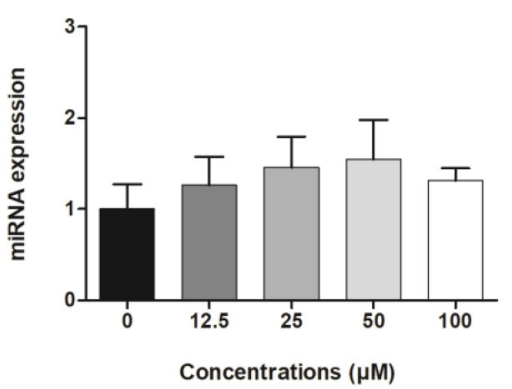

miR-145

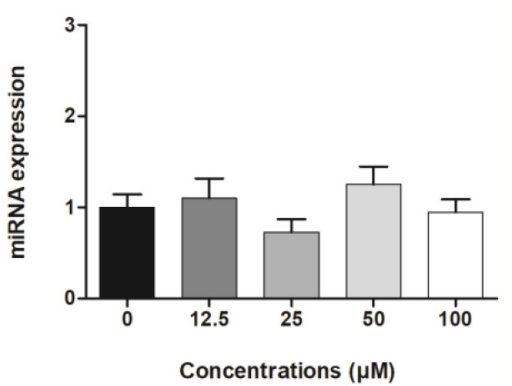

FIGURE 3 | Expression of exosomal miRNAs derived from HepG2 cells treated with clopidogrel. Cells were treated with clopidogrel (6.25, 12.5, 25, 50, and 100 $\mu$ M) for $24 \mathrm{~h}$ and $48 \mathrm{~h}$. Data are shown as fold change of normalized relative quantities (NRQ) of clopidogrel-treated cells divided by control cells (vehicle, $0 \mu \mathrm{M}$ clopidogrel), and analyzed by Kruskal-Wallis ANOVA and pairwise comparisons by Mann-Whitney test. * $p<0.05$ compared to control. Data from 3 independent experiments were performed in duplicate. 
EIF4G2

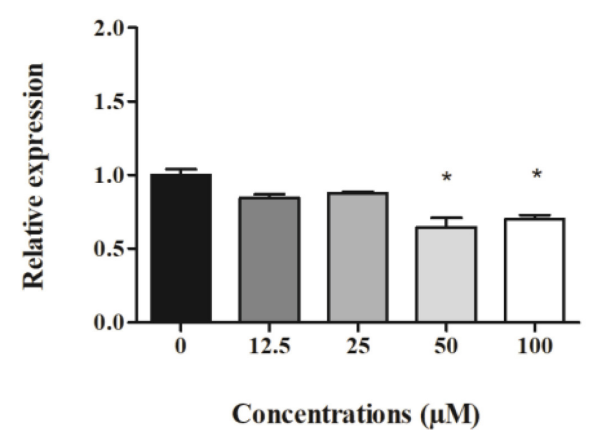

PLOD2

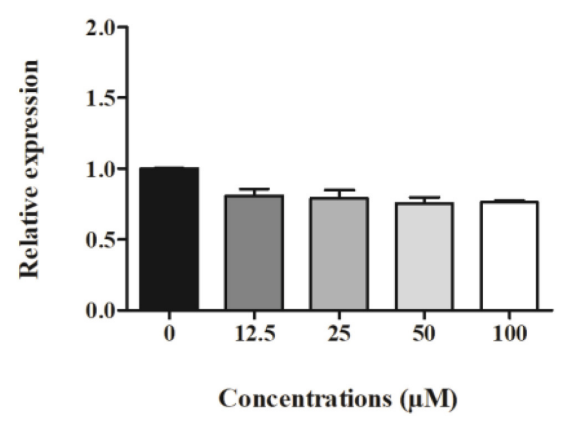

SENP5

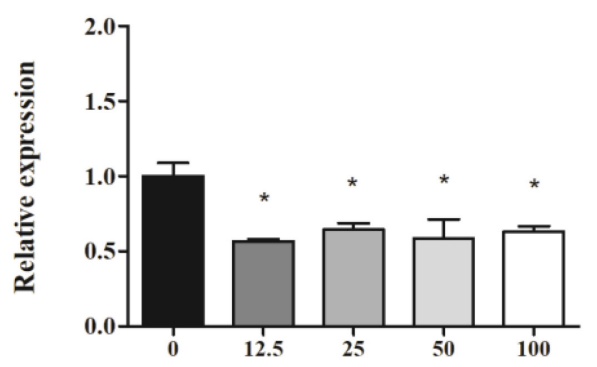

Concentrations $(\mu \mathrm{M})$
HMGA2

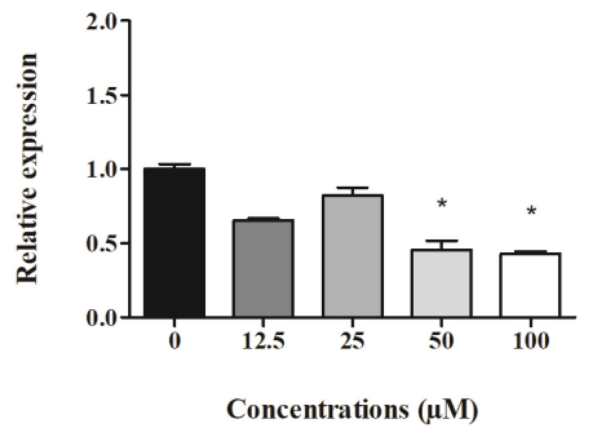

STRADB

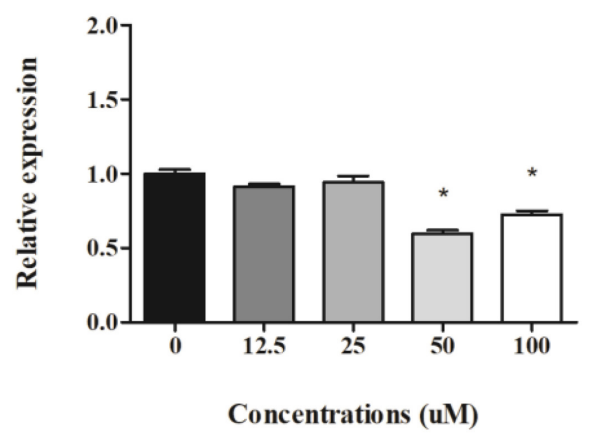

TLK1

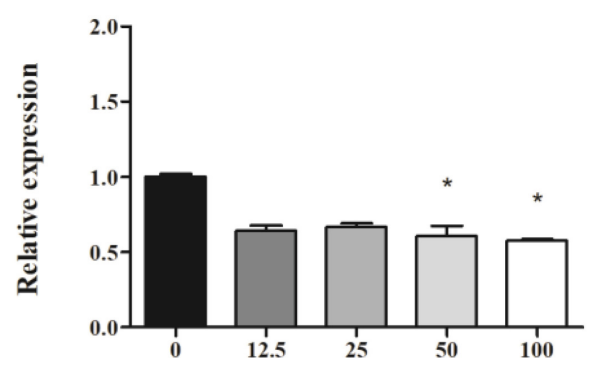

Concentrations $(\mu \mathrm{M})$

FIGURE 4 | Expression of cell-derived mRNAs in HepG2 cells after $24 \mathrm{~h}$ treatment with clopidogrel (12.5, 25, 50, and 100 $\mu$ M). Data are shown as fold change $\left(2^{-\Delta \Delta \mathrm{Ct}}\right)$ of clopidogrel-treated cells, using the vehicle $(0 \mu \mathrm{M}$ clopidogrel), as control and compared by Kruskal-Wallis ANOVA and pairwise comparisons by Mann-Whitney test. ${ }^{*} p<0.05$ compared to control. Data from three independent experiments performed in duplicate.

reported previously may be due to differences in the methodology used for cytotoxicity assessment compared to our study.

In HepG2 cells, CYPs have low activity, particularly CYP1A2, CYP2B6, and CYP3A4 (Gerets et al., 2012; O'Brien, 2014). The low expression levels of these CYP enzymes combined with the lack of CAM toxicity in HepG2 cells suggest that clopidogrel (pro-drug) itself can cause hepatocellular lesions. This is particularly important in patients with low CYP activity due to genetic variations (e.g., CYP2C19*2, poor metabolizers, who are exposed to high concentrations of clopidogrel than the CYP2C19*1, extensive metabolizers) or drug-drug interactions for both the standard- and high-dose regimens.

In this study, high doses of clopidogrel altered the exosomalderived miRNAs miR-26a and miR-15b. These miRNAs have been shown to be associated with liver regeneration, cell cycle control, cell growth, survival, development, DNA damage, and apoptosis. 
EIF4G2

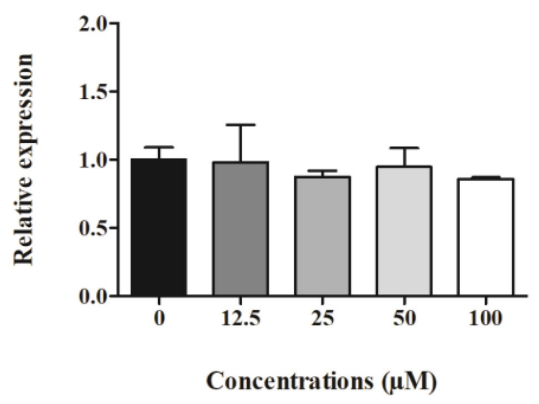

PLOD2

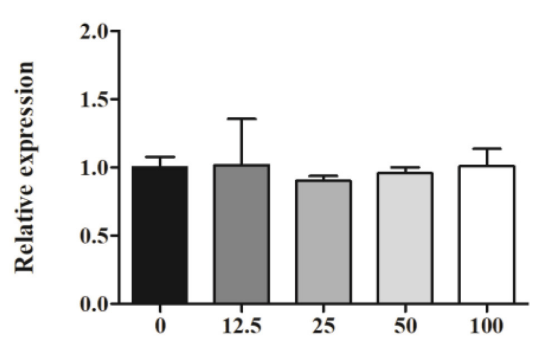

Concentrations $(\mu \mathrm{M})$

SENP5

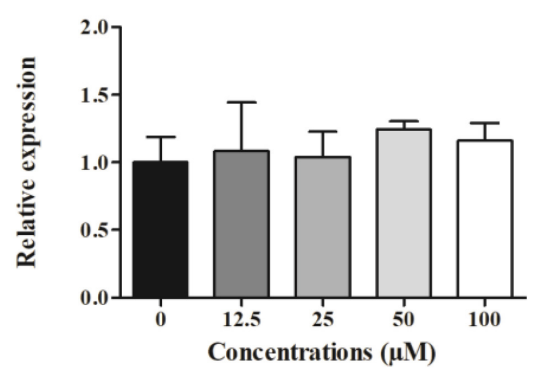

HMGA2

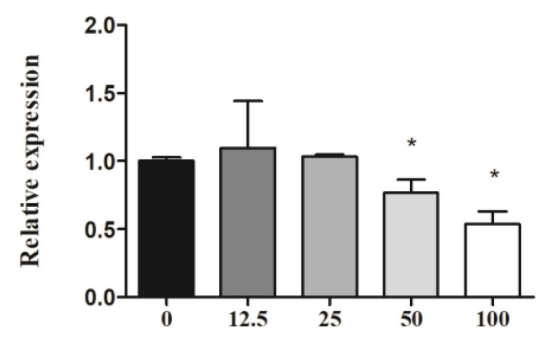

Concentrations $(\mu \mathrm{M})$

STRADB

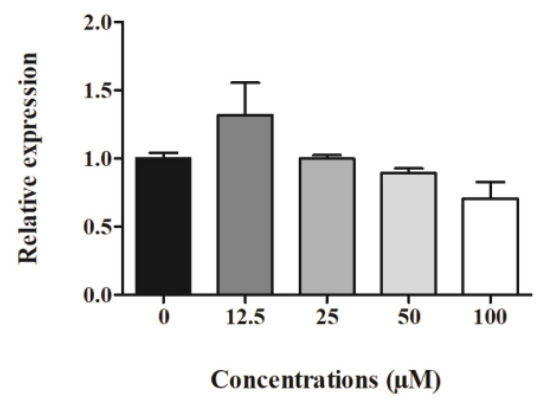

TLK1

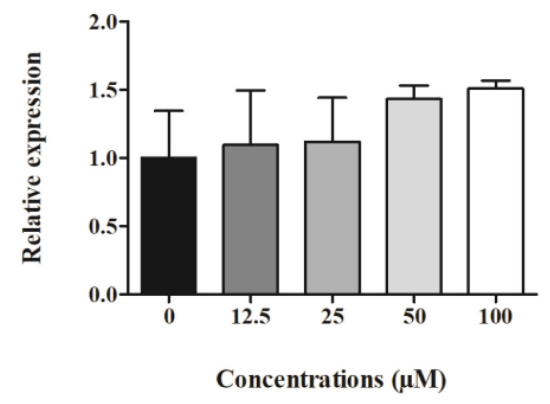

FIGURE 5 | Expression of cell-derived mRNAs in HepG2 cells after 48 h treatment with clopidogrel (12.5, 25, 50, and $100 \mu \mathrm{M})$. Data are shown as fold change $\left(2^{-\Delta \Delta \mathrm{Ct}}\right)$ of clopidogrel-treated cells, using the vehicle $(0 \mu \mathrm{M}$ clopidogrel), as control and compared by Kruskal-Wallis ANOVA and pairwise comparisons by Mann-Whitney test. ${ }^{*} p<0.05$ compared to control. Data from three independent experiments performed in duplicate.

In accordance with our previous in silico study, we observed upregulation of exosomal miR-26a levels following exposure to a cytotoxic concentration $(100 \mu \mathrm{M})$ of clopidogrel, which suggested that miR-26a upregulation may play an important role in the clopidogrel response and hepatotoxicity (Freitas et al., 2016). Likewise, increased miR-26a expression was also observed in a toxicological study of HepG2 cells using benzo[a]pyrene $(\mathrm{BaP})$, a polycyclic aromatic hydrocarbon, as the genotoxin/carcinogen. It has been suggested that miR-26a modulates important mRNA networks involved in toxicity, in response to $\mathrm{BaP}$ treatment (Lizarraga et al., 2012). Overexpression of the miR-26 family also resulted in an accumulation of the G1 population of HepG2 cells, through inhibition of cell cycle progression (Zhu et al., 2012). Conversely, downregulation of miR-26a induced hepatocyte proliferation during liver regeneration in male C57BL/6J mice (Zhou et al., 2012).

In this study, the cell cycle profile of HepG2 cells treated with toxic concentrations ( 50 and $100 \mu \mathrm{M}$ ) of clopidogrel may be caused by a combination of apoptosis and the effect of miR-26 on cell cycle control.

In this study, the expression levels of the miR-26a mRNA targets HMGA2, STRADB, EIF4G2, and SENP5 in HepG2 cells were significantly downregulated following exposure to a toxic concentration $(100 \mu \mathrm{M})$ of clopidogrel. These genes are involved with general cellular processes, such as cell 
TABLE 1 | miRNAs-mRNAs regulatory pathways.

\begin{tabular}{|c|c|c|c|c|c|}
\hline miRNA & Change & target mRNA & Change & Regulatory pathway & References \\
\hline \multirow[t]{5}{*}{ miR-26a-5p } & Upregulation & & & $\begin{array}{l}\text { Liver regeneration, cell cycle control, } \\
\text { apoptosis }\end{array}$ & $\begin{array}{l}\text { Lizarraga et al., 2012; Zhou et al., 2012; Zhu } \\
\text { et al., 2012; Freitas et al., } 2016\end{array}$ \\
\hline & & HMGA2 & Downregulation & Cell growth and development & Sun et al., 2013; Zhou et al., 2014 \\
\hline & & EIF4G2 & Downregulation & DNA damage and apoptosis & Badura et al., 2012 \\
\hline & & STRADB & Downregulation & Cell cycle control & Zhong et al., 2013 \\
\hline & & SENP5 & Downregulation & Cell growth and apoptosis & Di Bacco et al., 2006; Wang and Zhang, 2014 \\
\hline \multirow[t]{2}{*}{$\operatorname{miR}-15 b-5 p$} & Downregulation & & & Cell survival and cell cycle control & van Rooij et al., 2012 \\
\hline & & $T L K 1$ & Downregulation & Cell cycle control & Kim et al., 2016; Timiri Shanmugam et al., 2016 \\
\hline
\end{tabular}

Regulatory pathways were obtained from data published in National Center for Biotechnology Information (NCBI PubMed; https://www.ncbi.nlm.nih.gov/pubmed/).

growth and development, cell cycle control, DNA damage, and apoptosis.

HMGA2 encodes high mobility group at-hook 2, which is an architectural protein involved in various cellular processes, such as uncontrolled growth and development and alteration of chromatin architecture, that acts by modifying the interaction of transcriptional enhancers (Sun et al., 2013). A previous study showed that HMGA2 mRNA levels were inversely correlated with miR-26a expression and that reduced levels of HMGA2 inhibited the proliferation of gallbladder cancer cells (Zhou et al., 2014). Therefore, downregulation of HMGA2 may be involved in the cytotoxic mechanism of clopidogrel in HepG2 cells, by affecting cell growth and development.

STRADB encodes STE20-related kinase adaptor beta, which is involved in the induction of cell cycle arrest by controlling the relocation of serine/threonine protein kinase 11 (STK11 or LKB1), from the nucleus to the cytoplasm (Zhong et al., 2013). It is likely that reduced expression of STRADB is associated with increased cell cycle length and consequent slowing down of the cell cycle, especially at G1 phase. This hypothesis is supported by the DNA fragmentation and cell cycle data collected using HepG2 cells exposed to toxic concentrations of clopidogrel.

Eukaryotic translation initiation factor 4-gamma 2 is encoded by EIF4G2. It has been shown that silencing of EIF4G2 increases the DNA damage induced by ionizing radiation in breast cancer cells (Badura et al., 2012). Moreover, reduced levels of EIF4G2 have been suggested to be involved in apoptosis (Signoretti et al., 2010). It is likely that downregulation of EIF4G2 led to the increased DNA fragmentation and apoptosis observed in response to clopidogrel. This cytotoxic mechanism may be associated with an accumulation of reactive oxygen species and mitochondrial damage in the presence of the clopidogrel (Zahno et al., 2013; Zhai et al., 2016).

SENP5 encodes sentrin-specific protease 5, a C-terminal hydrolase that activates small ubiquitin-like SUMO proteins, which are involved in several biologic processes, including cell division and proliferation (Di Bacco et al., 2006). Inhibition of SENP5 was shown to suppress cell growth and induce apoptosis (Wang and Zhang, 2014). In this study, all tested concentrations of clopidogrel downregulated SENP5 mRNA levels; therefore, other mechanisms, besides the miR-26a pathway, may be involved in the regulation of SENP5 by clopidogrel.
PLOD2 is also a target mRNA of miR-26a. However, in this study, clopidogrel did not alter PLOD2 expression in HepG2 cells, which suggests that PLOD2 is not involved in the cytotoxic mechanism of clopidogrel.

Exosomal miR-15b was regulated by toxic concentrations $(100 \mu \mathrm{M})$ of clopidogrel after $48 \mathrm{~h}$ of treatment. Downregulation of miR-15b was also reported in TM4 cells treated with the cytotoxic xenobiotic nonylphenol for $24 \mathrm{~h}$ (Choi et al., 2011) and in cardiomyocytes derived from human pluripotent stem cells exposed to doxorubicin (Holmgren et al., 2016). These results suggest that suppression of miR-15b may be involved in the cytotoxic mechanism of clopidogrel and other drugs. Although the exact functions of miR-15b are still being explored, miR$15 \mathrm{~b}$ has been implicated in cell survival and cell cycle regulation under different clinical conditions, such as cancer and cardiac diseases (van Rooij et al., 2012). The role of miR-15b in liver cells has not been fully elucidated, and more studies are needed to investigate its regulatory effects in the cell survival and cell cycle control pathways.

We previously suggested that miR-15b may be a regulator of TLK1 (Freitas et al., 2016). In this study, miR-15b and TLK1 levels in HepG2 cells were downregulated by cytotoxic levels of clopidogrel. This result suggests that other molecules are involved in the modulation of TLK1 expression following exposure to clopidogrel. TLK1 encodes tousled-like kinase 1, a nuclear serine/threonine kinase that regulates chromatin assembly. TLK1 repression resulted in delayed S-phase progression in luminal breast cancers, suggesting its important role in cell cycle regulation (Kim et al., 2016). TLK1 was also involved in DNA damage-induced checkpoint arrest and suppressed cytotoxicity in salivary gland cells (Timiri Shanmugam et al., 2016). Therefore, TLK1 downregulation may be also involved in clopidogrel-induced hepatotoxicity, by controlling the cell cycle.

In this study, exosomal expression of miR-145 and miR-4701 was not affected by clopidogrel. miR- 145 has been associated with suppression of cell proliferation, invasion, and migration and induction of apoptosis in melanoma cells (Liu et al., 2017; Moon et al., 2017).

The mechanisms underlying clopidogrel-induced hepatotoxicity have not been fully elucidated. The general mechanisms of hepatotoxicity involve cellular accumulation 
of reactive oxygen species and opening of the mitochondrial permeability transition pore, leading to a release of cytochrome $\mathrm{c}$ into the cytoplasm, which is a key event in apoptosis (Zahno et al., 2013; Zhai et al., 2016), supporting our finding that clopidogrelinduced hepatotoxicity is directly related to apoptosis. Moreover, considering that this adverse effect is idiosyncratic (Kapila et al., 2015), understanding the regulatory mechanism will promote future studies attempting to validate the epigenetic mechanism involved in drug-induced hepatotoxicity.

In this study, high concentrations of clopidogrel led to dysregulated expression of exosomal miRNAs and target mRNAs in HepG2 cells. However, these effects were not related to changes in supernatant levels of AST and ALT, two markers of liver injury. It is likely that changes in exosomal miRNA expression are early events related to clopidogrel cell toxicity, and that dysregulation of miR-26a and miR-15b may be useful markers of drug-induced toxicity in clinical settings (Bronze-da-Rocha, 2014; Marrone et al., 2015; Yu et al., 2016).

It would be interesting to expand this analysis of cellular miRNA expression in clopidogrel-treated HepG2 cells to an investigation of the qualitative and quantitative distribution of miRNAs in source cells and their exosomes, which could provide more information regarding the contribution of circulating miRNAs for clinical evaluation of drug-induced liver injury.

In conclusion, toxic concentrations of clopidogrel modulate the expression of miR-26a and miR-15b and their mRNA targets EIF4G2, HMGA2, and STRADB in HepG2 cells, which regulate the cell cycle and apoptosis, mechanisms that maybe involved in clopidogrel-induced liver injury.

\section{REFERENCES}

Badimon, L., and Vilahur, G. (2014). Thrombosis formation on atherosclerotic lesions and plaque rupture. J. Intern. Med. 276, 618-632. doi: 10.1111/joim.12296

Badura, M., Braunstein, S., Zavadil, J., and Schneider, R. J. (2012). DNA damage and eIF4G1 in breast cancer cells reprogram translation for survival and DNA repair mRNAs. Proc. Natl. Acad. Sci. U.S.A. 109, 18767-18772. doi: 10.1073/pnas.1203853109

Bala, S., Petrasek, J., Mundkur, S., Catalano, D., Levin, I., Ward, J., et al. (2012). Circulating microRNAs in exosomes indicate hepatocyte injury and inflammation in alcoholic, drug-induced, and inflammatory liver diseases. Hepatology 56, 1946-1957. doi: 10.1002/hep.25873

Beitelshees, A. L., Voora, D., and Lewis, J. P. (2015). Personalized antiplatelet and anticoagulation therapy: applications and significance of pharmacogenomics. Pharmgenomics Pers. Med. 8, 43-61. doi: 10.2147/PGPM.S52900

Bouman, H. J., Schömig, E., van Werkum, J. W., Velder, J., Hackeng, C. M., Hirschhäuser, C., et al. (2011). Paraoxonase-1 is a major determinant of clopidogrel efficacy. Nat. Med. 17, 110-116. doi: 10.1038/nm.2281

Bronze-da-Rocha, E. (2014). MicroRNAs expression profiles in cardiovascular diseases. Biomed Res. Int. 2014:985408. doi: 10.1155/2014/985408

Caplain, H., Donat, F., Gaud, C., and Necciari, J. (1999). Pharmacokinetics of clopidogrel. Semin. Thromb. Hemost. 25(Suppl. 2), 25-28.

Chen, S., Qi, X., Chen, H., Li, M., Gu, J., Liu, C., et al. (2016). Expression of miRNA$26 \mathrm{a}$ in platelets is associated with clopidogrel resistance following coronary stenting. Exp. Ther. Med. 12, 518-524. doi: 10.3892/etm.2016.3278

Choi, J. S., Oh, J. H., Park, H. J., Choi, M. S., Park, S. M., Kang, S. J., et al. (2011). miRNA regulation of cytotoxic effects in mouse Sertoli cells exposed to nonylphenol. Reprod. Biol. Endocrinol. 9:126. doi: 10.1186/1477-7827-9-126

\section{AUTHOR CONTRIBUTIONS}

AnDL and VS were responsible for the original concept of the study, as well as the study design; RF, RB, ML, LT, and LM performed the experimental study and laboratory analyses; AnDL, VS, AuDL, MH, RH, RB, and RF interpreted the results and performed the statistical analyses; $\mathrm{RF}$ and $\mathrm{RB}$ wrote the manuscript; $\mathrm{AnDL}, \mathrm{AuDL}$, and $\mathrm{MH}$ were responsible for financial support; AnDL, AuDL, RH, and $\mathrm{MH}$ critically revised the manuscript.

\section{FUNDING}

This study was supported by grants from the Conselho Nacional de Desenvolvimento Científico e Tecnológico (CNPq) (448753/2014-6 and 447120/2014-0) and Fundação de Amparo à Pesquisa do Estado de São Paulo (FAPESP) (2010/18095-0).

\section{ACKNOWLEDGMENTS}

$\mathrm{MH}$ and $\mathrm{RH}$ are recipients of fellowships from CNPq, Brazil. $\mathrm{RF}$ and $\mathrm{RB}$ are recipient of fellowships from CAPES, Brazil. LM and LT were recipient of fellowship from FAPESP and CAPES (Brazil), respectively. We thank Karina Danielle Pereira for technical assistance.

\section{SUPPLEMENTARY MATERIAL}

The Supplementary Material for this article can be found online at: https://www.frontiersin.org/articles/10.3389/fphar. 2017.00906/full\#supplementary-material

Di Bacco, A., Ouyang, J., Lee, H. Y., Catic, A., Ploegh, H., and Gill, G. (2006). The SUMO-specific protease SENP5 is required for cell division. Mol. Cell. Biol. 26, 4489-4498. doi: 10.1128/MCB.02301-05

Enderle, D., Spiel, A., Coticchia, C. M., Berghoff, E., Mueller, R., Schlumpberger, M., et al. (2015). Characterization of RNA from exosomes and other extracellular vesicles isolated by a novel spin column-based method. PLoS ONE 10:e0136133. doi: 10.1371/journal.pone.0136133

Fintel, D. J. (2012). Oral antiplatelet therapy for atherothrombotic disease: overview of current and emerging treatment options. Vasc. Health Risk Manag. 8, 77-89. doi: 10.2147/VHRM.S26030

Fitzgerald, D. J., and Fitzgerald, G. A. (2013). Historical lessons in translational medicine: cyclooxygenase inhibition and P2Y12 antagonism. Circ. Res. 112, 174-194. doi: 10.1161/CIRCRESAHA.111.300271

Fontana, R. J. (2014). Pathogenesis of idiosyncratic drug-induced liver injury and clinical perspectives. Gastroenterology 146, 914-928. doi: 10.1053/j.gastro.2013.12.032

Freitas, R. C. C., Bortolin, R. H., Lopes, M. B., Hirata, M. H., Hirata, R. D. C., Silbiger, V. N., et al. (2016). Integrated analysis of miRNA and mRNA gene expression microarrays: influence on platelet reactivity, clopidogrel response and drug-induced toxicity. Gene 593, 172-178. doi: 10.1016/j.gene.2016.08.028

Gerets, H. H., Tilmant, K., Gerin, B., Chanteux, H., Depelchin, B. O., Dhalluin, S., et al. (2012). Characterization of primary human hepatocytes, HepG2 cells, and HepaRG cells at the mRNA level and CYP activity in response to inducers and their predictivity for the detection of human hepatotoxins. Cell Biol. Toxicol. 28, 69-87. doi: 10.1007/s10565-011-9208-4

Goyal, R. K., Srivastava, D., and Lessnau, K. D. (2009). Clopidogrelinduced hepatocellular injury and cholestatic jaundice in an elderly patient: case report and review of the literature. Pharmacotherapy 29, 608-612. doi: $10.1592 /$ phco.29.5.608 
Hayes, C. N., and Chayama, K. (2016). Micrornas as biomarkers for liver disease and hepatocellular carcinoma. Int. J. Mol. Sci. 17:280. doi: $10.3390 /$ ijms 17030280

Holmgren, G., Synnergren, J., Andersson, C. X., Lindahl, A., and Sartipy, P. (2016). MicroRNAs as potential biomarkers for doxorubicin-induced cardiotoxicity. Toxicol. In Vitro 34, 26-34. doi: 10.1016/j.tiv.2016.03.009

Huang, X., Yuan, T., Tschannen, M., Sun, Z., Jacob, H., Du, M., et al. (2013). Characterization of human plasma-derived exosomal RNAs by deep sequencing. BMC Genomics 14:319. doi: 10.1186/1471-2164-14-319

Kapila, A., Chhabra, L., Locke, A. D., Patel, P., Khanna, A., Reddy, C. M., et al. (2015). An idiosyncratic reaction to clopidogrel. Perm. J. 19, 74-76. doi: 10.7812/TPP/14-040

Kim, J. A., Tan, Y., Wang, X., Cao, X., Veeraraghavan, J., Liang, Y., et al. (2016). Comprehensive functional analysis of the tousled-like kinase 2 frequently amplified in aggressive luminal breast cancers. Nat. Commun. 7, 12991. doi: 10.1038/ncomms12991

Liu, S., Gao, G., Yan, D., Chen, X., Yao, X., Guo, S., et al. (2017). Effects of miR-145$5 p$ through NRAS on the cell proliferation, apoptosis, migration, and invasion in melanoma by inhibiting MAPK and PI3K/AKT pathways. Cancer Med. 6, 819-833. doi: 10.1002/cam4.1030

Livak, K. J., and Schmittgen, T. D. (2001). Analysis of relative gene expression data using real-time quantitative, PCR and the $2-\Delta \Delta$ CT Method. Methods 25, 402-408. doi: 10.1006/meth.2001.1262

Lizarraga, D., Gaj, S., Brauers, K. J., Timmermans, L., Kleinjans, J. C., and van Delft, J. H. (2012). Benzo[ a ]pyrene-induced changes in microRNA-mRNA networks. Chem. Res. Toxicol. 25, 838-849. doi: 10.1021/tx2003799

Maffrand, J. P. (2012). The story of clopidogrel and its predecessor, ticlopidine: could these major antiplatelet and antithrombotic drugs be discovered and developed today? Comptes. Rendus Chim. 15, 737-743. doi: 10.1016/j.crci.2012.05.006

Marabita, F., de Candia, P., Torri, A., Tegnér, J., Abrignani, S., and Rossi, R. L. (2016). Normalization of circulating microRNA expression data obtained by quantitative real-time RT-PCR. Brief. Bioinform. 17, 204-212. doi: 10.1093/bib/bbv056

Marrone, A. K., Beland, F. A., and Pogribny, I. P. (2015). The role for microRNAs in drug toxicity and in safety assessment. Expert Opin. Drug Metab. Toxicol. 11, 601-611. doi: 10.1517/17425255.2015.1021687

McGill, M. R., and Jaeschke, H. (2015). MicroRNAs as Signaling Mediators and Biomarkers of Drug- and Chemical-Induced Liver Injury. J. Clin. Med. 4, 1063-1078. doi: $10.3390 / \mathrm{jcm} 4051063$

Miyamoto, K., Seki, N., Matsushita, R., Yonemori, M., Yoshino, H., Nakagawa, M., et al. (2016). Tumour-suppressive miRNA-26a-5p and miR-26b-5p inhibit cell aggressiveness by regulating PLOD2 in bladder cancer. Br. J. Cancer 115, 354-363. doi: 10.1038/bjc.2016.179

Monteiro, P. H., Pinheiro, L. d. S., Alvoeiro, L., Lucas, M., and Victorino, R. M. (2011). Clopidogrel-induced liver failure. JRSM Short Rep. 2, 40-40. doi: 10.1258/shorts.2011.010111

Moon, S., Kim, D. K., and Kim, J. (2017). Apoptosis-related microRNA-145-5p enhances the effects of pheophorbide a-based photodynamic therapy in oral cancer. Oncotarget 8, 35184-35192. doi: 10.18632/oncotarget.17059

O’Brien, P. J. (2014). High-content analysis in toxicology: screening substances for human toxicity potential, elucidating subcellular mechanisms and in vivo use as translational safety biomarkers. Basic Clin. Pharmacol. Toxicol. 115, 4-17. doi: $10.1111 /$ bcpt.12227

Ochoa-Cortes, F., Liñán-Rico, A., Jacobson, K. A., and Christofi, F. L. (2014). Potential for developing purinergic drugs for gastrointestinal diseases. Inflamm. Bowel Dis. 20, 1259-1287. doi: 10.1097/MIB.0000000000000047

Sangkuhl, K., Klein, T. E., and Altman, R. B. (2010). Clopidogrel pathway. Pharmacogenet. Genomics 20, 463-465. doi: 10.1097/FPC.0b013e3283385420

Signoretti, S., Vagnozzi, R., Tavazzi, B., and Lazzarino, G. (2010). Biochemical and neurochemical sequelae following mild traumatic brain injury: summary of experimental data and clinical implications. Neurosurg. Focus 29:E1. doi: 10.3171/2010.9.FOCUS10183

Stroynowska-Czerwinska, A., Fiszer, A., and Krzyzosiak, W. J. (2014). The panorama of miRNA-mediated mechanisms in mammalian cells. Cell. Mol. Life Sci. 71, 2253-2270. doi: 10.1007/s00018-013-1551-6
Sun, M., Song, C. X., Huang, H., Frankenberger, C. A., Sankarasharma, D., Gomes, S., et al. (2013). HMGA2/TET1/HOXA9 signaling pathway regulates breast cancer growth and metastasis. Proc. Natl. Acad. Sci. U.S.A. 110, 9920-9925. doi: 10.1073/pnas.1305172110

Sunderland, N., Skroblin, P., Barwari, T., Huntley, R. P., Lu, R., Joshi, A., et al. (2017). MicroRNA Biomarkers and Platelet Reactivity: the Clot Thickens. Circ. Res. 120, 418-435. doi: 10.1161/CIRCRESAHA.116.309303

Timiri Shanmugam, P. S., Nair, R. P., De Benedetti, A., Caldito, G., Abreo, F., and Sunavala-Dossabhoy, G. (2016). Tousled kinase activator, gallic acid, promotes homologous recombinational repair and suppresses radiation cytotoxicity in salivary gland cells. Free Radic. Biol. Med. 93, 217-226. doi: 10.1016/j.freeradbiomed.2015.12.029

van Rooij, E., Purcell, A. L., and Levin, A. A. (2012). Developing MicroRNA therapeutics. Circ. Res. 110, 496-507. doi: 10.1161/CIRCRESAHA.111.247916

Vickers, A. E., Ulyanov, A. V, and Fisher, R. L. (2017). Liver effects of clinical drugs differentiated in human liver slices. Int. J. Mol. Sci. 18:E574. doi: $10.3390 /$ ijms 18030574

Wang, K., and Zhang, X. C. (2014). Inhibition of SENP5 suppresses cell growth and promotes apoptosis in osteosarcoma cells. Exp. Ther. Med. 7, 1691-1695. doi: $10.3892 /$ etm.2014.1644

Weiler, S., Merz, M., and Kullak-Ublick, G. A. (2015). Drug-induced liver injury: the dawn of biomarkers? F1000Prime Rep. 7:34. doi: 10.12703/P7-34

Yang, Y., Lewis, J. P., Hulot, J. S., and Scott, S. A. (2015). The pharmacogenetic control of antiplatelet response: candidate genes and CYP2C19. Expert Opin. Drug Metab. Toxicol. 11, 1599-1617. doi: 10.1517/17425255.2015.1068757

Yu, X., Odenthal, M., and Fries, J. W. (2016). Exosomes as miRNA carriers: formation-function-future. Int. J. Mol. Sci. 17:E2028. doi: $10.3390 /$ ijms 17122028

Zahno, A., Bouitbir, J., Maseneni, S., Lindinger, P. W., Brecht, K., and Krähenbühl, S. (2013). Hepatocellular toxicity of clopidogrel: mechanisms and risk factors. Free Radic. Biol. Med. 65, 208-216. doi: 10.1016/j.freeradbiomed.2013.06.007

Zahno, A., Brecht, K., Bodmer, M., Bur, D., Tsakiris, D., A., and Krähenbühl, S. (2010). Effects of drug interactions on biotransformation and antiplatelet effect of clopidogrel in vitro. Br. J. Pharmacol. 161, 393-404. doi: 10.1111/j.1476-5381.2010.00881.x

Zhai, Y., Wang, L., Yang, F., Feng, G., Feng, S., Cui, T., et al. (2016). The mechanism and risk factors of clopidogrel-induced liver injury. Drug Chem. Toxicol. 39, 367-374. doi: 10.3109/01480545.2015.1122606

Zhang, J., Li, S., Li, L., Li, M., Guo, C., Yao, J., et al. (2015). Exosome and exosomal microRNA: trafficking, sorting, and function. Genomics Proteomics Bioinform. 13, 17-24. doi: 10.1016/j.gpb.2015.02.001

Zhong, D. S., Sun, L. L., and Dong, L. X. (2013). Molecular mechanisms of LKB1 induced cell cycle arrest. Thorac. Cancer 4, 229-233. doi: 10.1111/1759-7714.12003

Zhou, H., Guo, W., Zhao, Y., Wang, Y., Zha, R., Ding, J., et al. (2014). MicroRNA-26a acts as a tumor suppressor inhibiting gallbladder cancer cell proliferation by directly targeting HMGA2. Int. J. Oncol. 45, 2050-2058. doi: $10.3892 /$ ijo.2014.2360

Zhou, J., Ju, W., Wang, D., Wu, L., Zhu, X., Guo, Z., et al. (2012). Downregulation of microRNA-26A promotes mouse hepatocyte proliferation during liver regeneration. PLoS ONE 7:e33577. doi: 10.1371/journal.pone.0033577

Zhu, Y., Lu, Y., Zhang, Q., Liu, J. J., Li, T. J., Yang, J. R., et al. (2012). MicroRNA$26 \mathrm{a} / \mathrm{b}$ and their host genes cooperate to inhibit the G1/S transition by activating the pRb protein. Nucleic Acids Res. 40, 4615-4625. doi: 10.1093/nar/gkr1278

Conflict of Interest Statement: The authors declare that the research was conducted in the absence of any commercial or financial relationships that could be construed as a potential conflict of interest.

Copyright (๐ 2017 Freitas, Bortolin, Lopes, Tamborlin, Meneguello, Silbiger, Hirata, Hirata, Luchessi and Luchessi. This is an open-access article distributed under the terms of the Creative Commons Attribution License (CC BY). The use, distribution or reproduction in other forums is permitted, provided the original author (s) or licensor are credited and that the original publication in this journal is cited, in accordance with accepted academic practice. No use, distribution or reproduction is permitted which does not comply with these terms. 